\title{
Genetic Alteration of Selection Resistance on BAC-EBV Plasmids by Homologous Recombination and Establishment of the Stably Transfected Cell Line
}

\author{
Lielian ZUO, Meijuan ZHU, Shujuan DU, Qiao LI, Guiyuan LI, Jianhong LU* \\ Cancer Research Institute, Xiangya School of Medicine, Central South University; Changsha 410078, China
}

Liming HE

Newlife BioChemEx LLC, Bethesda, MD, USA

*Corresponding author: jianhlu@csu.edu.cn

\begin{abstract}
To obtain recombinant Epstein-Barr virus (EBV) particles with the neomycin resistance from BAC-EBV with hygrymicin (hyg) gene, homologous recombination was employed for altering the original hyg gene to neo resistance gene in the BAC-EBV genome. The kanamycin marker gene (kan) containing the recombinant protein FLP recognition sites (FRTs) was connected to the neo gene in the vector of pGEM-T using conventional cloning techniques. Both ends of the connective are the arms with $20 \mathrm{bp}$ of homology sequences responding to the ends next to the hyg gene. PCR and cloning techniques were further performed to extend homology arms to make them reach $89 \mathrm{bp}$. The linear fragment kan-neo with 89bp of arms was then electroporated into the bacteria containing BAC-EBV genome. Under the mediation of the red $\alpha \beta \gamma$ system, the hyg gene in BAC-EBV was replaced by neo gene. A series of methods including antibiotic selection, PCR amplification, sequencing and restriction enzyme digestion were used for the identification of the mutated plasmids. The results showed that BAC-EBV/neo, was constructed as expected. Through the transfection of the BAC-EBV/neo plasmid, G418 selection, and GFP observation, we established 293-EBV/neo cell line. This research lays a foundation for the establishment of broader cell models in the study of EBV-associated pathogenic mechanisms.
\end{abstract}

KEY WORDS: Epstein-Barr virus; homologous recombination; BAC; GFP

\section{INTRODUCTION}

EB virus (Epstein-Barr virus, EBV) is associated with kinds of human tumors, such as nasopharyngeal cancer and Burrkitt lymphoma[1-2]. Due to the large genome with $172 \mathrm{~kb}, \mathrm{EBV}$ is not easy for the genetic manipulation during the viral study in the context of the whole genome. The BAC-EBV is abacterial artificial chromosome (BAC)-based plasmid that contains the complete Epstein-Barr virus (EBV) large genome of $172 \mathrm{~kb}$, the E.Coli $\mathrm{F}$ factor, selection markers and GFP.[3] the E. Coli F factor as used in the bacterial artificial chromosome (BAC) vector was introduced into the genome of EBV. The $\mathrm{F}$ factor ensures the genome replication with a lowcopy number (1 copy) in bacteria strictly. BACbased EBV genome can accurately replicates with the BAC's replication, and is possible for the genetic modification in bacteria using the homologous recombination. In previously studies, recombinant virus particles from BAC-EBV plasmid infected the nasopharyngeal epithelial cells (NPC) in a low level of efficiency, and the strong sensitivity of the NPC to hygromycin(hyg) leads to the difficulty for the the stable selection. Thus, we alter the original hyg gene to neo resistance gene (neo) in the BAC-EBV genome by the homologous recombination under the red $\alpha \beta \gamma$ system. And establish the stable transfected 293 cell line. This cell line will easy to get recombinant EB virus particles with the neo resistance, and lays a foundation for the establishment of cell models in the study of EBVassociated NPC (nasopharyngeal carcinoma).

\section{MATERIALS AND METHODS}

\subsection{Cell types and plasmids}

293 was preserved and propagated in our laboratory, cultured in DMEM (GIBCO) supplemented with $10 \%$ fetal bovine serum, $5 \% \mathrm{CO} 2$ atmosphere at $37^{\circ} \mathrm{C}$. Cells were collected during the exponential growth phase. DMEM (Dulbecco's Modified Eagle's medium) purchased from Hgclone company; pKD46,pKD13,pCP20 were the gifts from E. coli 
Genetic Stock enter (CGSC), Department of Molecular and Cellular Development Biology, Yale University. BAC-EBV was kindly providely by prof.Dr.W.Hammerschmid [3-4].

\subsection{PCR amplification to the target linear fragments}

The kan gene fragment containing FRT sites was connected to the vector pcDNA3.1(+) in previous studies[4]. This fragment upstream includes the homology gene with 20bp and restriction enzyme cutting site, BamHI, locates in the fragment downstream. PCR amplified the neo gene through the designed specific primer. And the neo fragment upstream was introduced into the BamHI site to easy to connect the kan gene fragment. The neo gene fragment downstream also contained 20bp homology next to hyg gene. The connective fragment "-arm1kan-neo-arm2" (Figure.1) was cloned into the plasmid pGEM-T easy vector to twice extend the homogous rams reach to $89 \mathrm{bp}$. The neo primers: the kan primers: P-kan-F, 5'-CAGTCTCAGGCCCC CTCACTTTGGGTCTTAAGGCTGGAGCTGCTT CGAA-3' and P-kan-R, 5'- TCTGGATCCGCA TCGATGGCCCGTGCGGCCATCCGTCGACCTG CAGTTCG-3'. The twice extend homologous primers: Extension 1-F, 5'-CGCGACCCAGCCGAGC GAGCTTAGCGAACTGTGGACGAGA-3'; Extension 1-R， 5'-TTCCAGAAGTAGTGAGGAGGCTTTTT TGGAGGCCTAGGCT-3'; Extension 2-F, 5'-ATCA CTGCGACCGGCCCTCCCGCGACCCAGCCGA GCGAGC-3'; Extension 2-R, 5'-CCTCCTCACTA CTTCTGGAATAGCTCAGAGGCCGAGGCGG-3'.

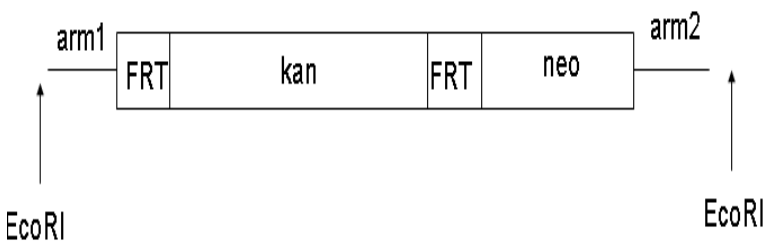

Figure 1: The construct of the linear fragment

\subsection{Electroporation of the target linear fragment, removal of the kanamycin resistance gene and identification of the recombinants}

Accroding to the method of Zhengyuan $\mathrm{Yu}$, Jianhong Lu, Haibo Yu.2011 [3], prepare the eleccompetent cells including pKD46 and BAC-EBV induced by newly prepared L-arabinose at a final concentration of $0.1 \%(\mathrm{w} / \mathrm{v})$.Then the cells expressed the proteins Red $\alpha, \beta$ and $\gamma$ to the electrotransformation. The 300ng target linear fragment was electroporated into 100ul of newly prepared competent bacterial cells in a $1 \mathrm{~mm}$ cuvette, using a gene pluser (ECM399,BT× Harvard Apparatus) at $1.7 \mathrm{kV}, 36 \mathrm{uF}, 5 \mathrm{~ms}$.the cells were grown on the $\mathrm{LB}$ plates $(\mathrm{Cam}+, \mathrm{Kan}+)$ at $30^{\circ} \mathrm{C}$. Then, the clones with both Cam and Kan resistance (Cam+, $\mathrm{Kan}+)$ were purified on LB plates at $42{ }^{\circ} \mathrm{C}$ for the removal of the pKD46 plasmid with Amp resistance. Through different resistances screened the positive clones with the Cam and Kan resistance without Amp resistance. Thus, the recombinants have the Cam and Kan resistance, and the positive bacterias have the Cam and Kan resistance without Amp resistance.

PCP20 was transferred into the before-mentioned positive clones induced by conventional preparative methods of competent cells. The kan gene was sitespecific excised by pcp20 expressive the Flp/FRT system. Then the pCP20 plasmid was removed at 42 -C.PCR identified the positive clones (BAC$\mathrm{EBV} / \mathrm{neo})$.

\subsection{Established of BAC-EBV/neo stable transfected 293 cell line}

Accroding to the manufacturer's introductions of the Lipofectamine ${ }^{\circledR}$ Transfection Reagent from Invitrogen Company, plasmid EBV-EBV/neo was transfected into 293 in a $50 \mathrm{~mm}$ culture flask. After 48 hours, the cells were selected by different levels of G418 (400 mg/ml, $600 \mathrm{mg} / \mathrm{ml}, 800 \mathrm{mg} / \mathrm{ml}, 1000$ $\mathrm{mg} / \mathrm{ml})$.

A fluorescence microscope was used to observe the GFP expression.

\section{RESULTS AND DISCUSSION}

\subsection{Genetic Alteration of Selection Resistence on BAC-EBV Plasmids by Homologous Recombination}

The target linear fragment (arm1-kan-neo-arm2) was cloned to $\mathrm{T}$ vector. EcoRI restriction enzyme digested the $\mathrm{T}$ vector, The results, shown in Figure 2 , indicate that the linear fragment was constructed successfully. And the constructed $\mathrm{T}$ vector was sequenced to analyze the correctness of the linear fragment.

\section{DL2000}

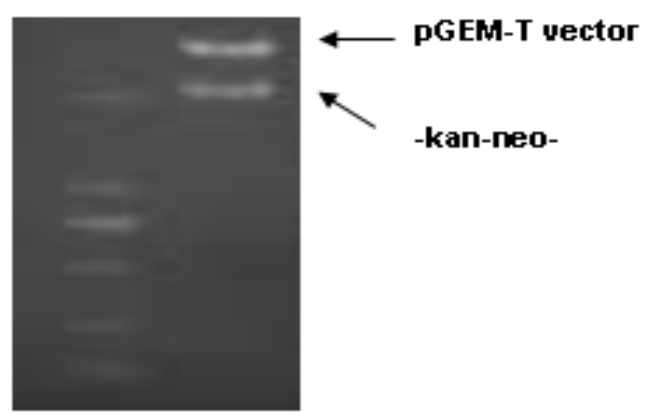

Figure 2: Identification of the $\mathrm{T}$ vector with the target linear fragment by EcoRI restriction enzyme. DL2000:2kb DNA ladder; right lane: pGEM-T vector and the linear fragment (arm1-kan-neo- arm2). 
BAC-EBV, contains EBV genome $172 \mathrm{~kb}$ from the B95-8, screening of molecular markers and GFP expression element. Because of the large EBVgenome, the conventional enzymatic digestion and connection technology can not introduce mutations in the plasmid BAC-EBV. Homologous recombination technology can solve this problem. This study used the method combined with the appropriate clone methods, successfully replace the hyg gene of the plasmid BAC-EBV .The homologous recombination involves two kinds of the important plasmids, respectively pKD46, pKD13 and pCP20. PKD46 can express the red $\alpha \beta \gamma$ system to mediate the homologous recombination. Plasmid pCP20 is able to expresse the recombinant protein FLP to specially recognize the FRT sites, so as to cut off the Kan gene, leaving the "Scar" series69bp.Those plasmids are the temperature sensitive type, and have Amp resistance, when cultured at a $42{ }^{\circ} \mathrm{C}$, will automatically lose.

After the linear fragment electrotransformed, the positive clones were selected through the different resistance, which have the $\mathrm{Cam}+$ and $\mathrm{Kan}+$ resistance. Then $\mathrm{pCP} 20$ was transferred into the competent cells of the positive clones. The kan gene was site-specific excised by pcp20 expressive the Flp/FRT system, leaving a FRT "scar" of $69 \mathrm{bp}$ showm in Figure 3(A). PCR identified further the positive clones(BAC-EBV/neo) shown in Figure 3(B), and the plasmid BAC-EBV/neo has the Cam+ resistance without Kan+ resistance.

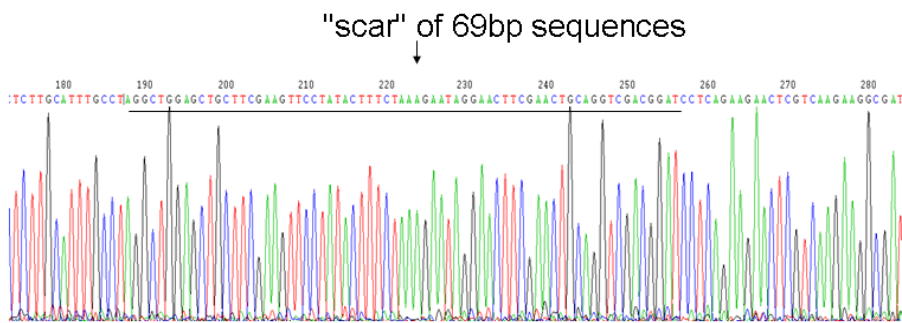

(A)

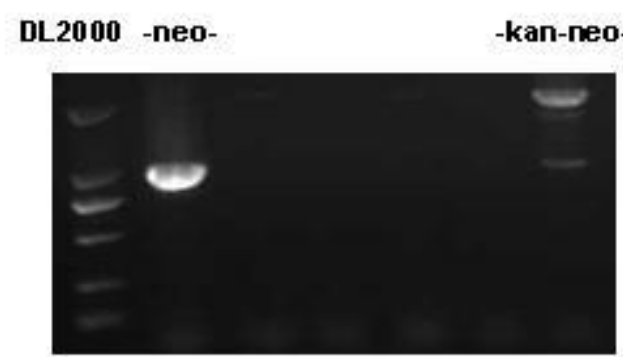

(B)

Figure 3: PCR-identification of targeted clones after removal of the kanamycin resistance gene. (A) The confirmation of the recombination site including the 69bp scar by sequencing. (B) PCR-identification of 4 clones after the removal of the kan gene. DL2000:2kb DNA ladder; Lane -neo- represent the removal of the kan gene of the positive clone; Lane -kan-neorepresent a control.

\subsection{Establishment of the Stably transfected Cell Line}

The plasmid BAC-EBV/neo was transfected into 293 cells. Through selection of the different concentrations of G418, such as $400 \mathrm{mg} / \mathrm{ml}$, $600 \mathrm{mg} / \mathrm{ml}, 800 \mathrm{mg} / \mathrm{ml}$ and $1000 \mathrm{mg} / \mathrm{ml}$. The $293-$ p2089/neo cell line was stable established successfully. The result is shown in Figure 5. More than $95 \%$ of the cells expressed the GFP.GFP expression makes the virus readout convenient and intuitive. GFP has been used in various fields, such as observation of the expression of specific genes, tumor angiogenesis, tumor growth, invasion and metastasis, and curative effect in cancer research and so on [5-7]. Establishment of the cell line stably transfected with plasmid BAC-EBV/neo provides the cell model of the research of the virus pathogenic mechanisms.

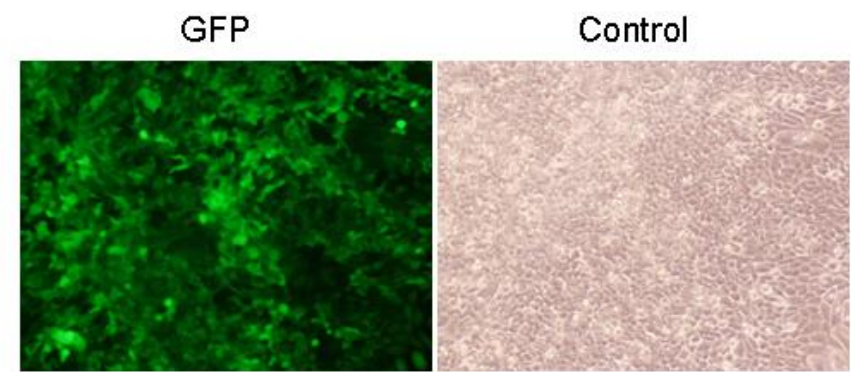

Figure 4: The derived cell line stably transfected with EBV genome (BAC-EBV/neo). The cell line 293-p2089/neo showed EGFP expression under an inverted fluorescence microscopy. The left picture showed the green fluorescence; the right picture was the contrast of the same field as a white light control.

\subsection{Conclusions}

Homologous recombination is applied to the genetic manipulation for a large genome. This study has used the method to construct the plasmid BAC$\mathrm{EBV} /$ neo on based of plasmid BAC-EBV. And then, establish the 293-EBV/neo cell line stably transfected BAC-EBV/neo. It will provide the object of the researches of the viral pathogenic mechanisms.

\section{ACKNOWLEDGEMENTS}

This work was supported by the projects of National Natural Science Foundation of China (81171931, 81372139) and Hunan Provincial Natural Science Foundation of China (2015JJ2149).

\section{REFERENCES}

[1] Maeda, E., et al., Spectrum of Epstein-Barr virus-related diseases: a pictorial review. Jpn J Radiol, 2009. 27(1): 419. 
[2] Wei, W.I. and J.S. Sham, Nasopharyngeal carcinoma. Lancet, 2005. 365(9476): 2041-54.

[3] Zhengyuan Yu, Jianhong Lu, Haibo Yu, Qijia Yan, Lielian Zuo, Jin Huang, Guiyuan Li. A precise one-time excision of the complete Epstein-Barr virus genome in a plasmid based on a bacterial artificial chromosome. J Virol Method, 2011, 176:103-107.

[4] Jianhong Lu, Yunlian Tang, Ming Zhou, Minghua Wu, Jue Ouyang, Gene modification in the genome of EpsteinBarr virus cloned as a bacterial artificial chromosome. Acta Microbiologica Sinica 2008, 48(3): 385-390.
[5] Tsai M H, Aki R, Amoh Y, et al. GFP-fluorescence-guided UVC irradiation inhibits melanoma growth and angiogenesis in nude mice. Anticancer Res, 2010, 30(9): 3291-3294.

[6] Castano A P, Liu Q, Hamblin M R. A green fluorescent protein-expressing murine tumour but not its wild-type counterpart is cured by photodynamic therapy. Br J Cancer, 2006, 94(3):391-397.

[7] Zagzag D, Miller D C, Chiriboga L, et al. Green fluorescent protein immunohistochemistry as a novel experimental tool for the detection of glioma cell invasion in vivo. Brain Pathol, 2003, 13(1):34-37. 